\title{
Observation of harmonically related solar radio zebra patterns in the 1-4 GHz frequency range
}

\author{
H. S. Sawant ${ }^{1}$, M. Karlický ${ }^{2}$, F. C. R. Fernandes ${ }^{1}$, and J. R. Cecatto ${ }^{1}$ \\ 1 Instituto Nacional de Pesquisas Espaciais, INPE, CP 515, 12201-970 São José dos Campos, SP, Brazil \\ 2 Astronomical Institute of the Academy of Sciences of the Czech Republic, 25165 Ondřejov, Czech Republic
}

Received 12 March 2002 / Accepted 24 September 2002

\begin{abstract}
A unique case of two zebra patterns related harmonically with ratio of $\sim 1: 2$ was observed by distant radio telescopes at São José dos Campos and Ondřejov Observatories. Accompanied zebras show that the ratio of frequencies of the neighboring zebra lines is in the range of 1.009-1.037. There is a tendency of a decrease of this ratio with decreasing frequency within the specific zebra pattern. Both facts speak in favour of plasma emission models for the zebra pattern fine structure in radio burst continua.
\end{abstract}

Key words. plasmas - Sun: flares - Sun: radio radiation

\section{Introduction}

The zebra patterns belong to fine structures of radio continua observed during solar flares. At metric wavelengths they have been known for a long time (e.g. Slottje 1972; Aurass \& Chernov 1983). Rosenberg (1972) and later Zheleznyakov \& Zlotnik (1975) modelled these structures in terms of the harmonics of the electron cyclotron modes. A similar model was suggested also by Kuijpers (1975). Other models (e.g. Chernov 1990) explained zebras by modulation of whistler packets.

With new radiospectrographs operating in the $\mathrm{GHz}$ frequency range the zebra patterns were discovered also in the 1-4 GHz range (Isliker \& Benz 1994). These highfrequency zebras are relatively rare (Jiřička et al. 2001). Therefore their observation and comparison with models is important.

In the present paper, first, examples of high-frequency zebra patterns observed by both Brazilian Solar Spectroscope (BSS) (Sawant et al. 2001) and Ondřejov radiospectrograph during the June 6, 2000 flare are presented. Among them a unique case of harmonically related zebra patterns observed at distant sites is shown. Using the plasma emission model of Ledenev et al. (2001) these zebras are analysed and magnetic fields in radio sources are estimated.

\section{The 1.2-4.5 GHz zebra pattern observations}

The June 6, 2000 flare classified as X2.3 was observed at 15:00-17:00 UT in the active region NOAA AR 9026.

Send offprint requests to: H. S. Sawant, e-mail: guga@das .inpe.br
A full-halo coronal mass ejection and type II/IV bursts were reported in association with this flare (Solar Flare NOAA Report).

This flare was rich in high-frequency zebra patterns. A global overview of observations of these zebras in comparison with the $3 \mathrm{GHz}$ radio flux record is shown in Fig. 1. Here it is shown that the zebra patterns were recorded after the first flare maximum and close to the secondary one. Parameters of the zebra patterns in the $1.2-1.7$ and $2.0-4.5 \mathrm{GHz}$ frequency ranges are given in Tables 1 and 2, respectively. The number of observed zebra lines is in the interval of 2-8. The modulation depth $Q$ of zebras (the ratio of the maximum to minimum radio flux) is listed in the last columns of Tables 1 and 2. Examples of the zebra patterns in the $1.2-1.7 \mathrm{GHz}$ and $2.0-4.5 \mathrm{GHz}$ ranges and the corresponding frequency cuts are shown in Figs. 2 and 3 , respectively.

The zebra patterns in the harmonic ratio of $\sim 1: 2$ are presented in Fig. 4. In both the $1.2-1.7 \mathrm{GHz}$ as well as the $2.0-4.5 \mathrm{GHz}$ range some zebra lines appear with varying intensity, simultaneously, and at frequencies in fundamental (F)/ harmonic $(\mathrm{H})$ ratio within the accuracy of the receivers (about $\pm 20 \mathrm{MHz}$ ). The zebra lines in the fundamental band are more intense than those in the harmonic one. Furthermore, the zebra lines in the fundamental band are shorter in time; after 15:37:45 UT only zebra lines in the harmonic band were observed. A detailed comparison of zebra frequencies at 15:37:43.5 UT shows zebra lines in the fundamental mode at 1620,1640 , and $1680 \mathrm{MHz}$. In the second harmonic mode, stripes are situated at $3200 \mathrm{MHz}(2 \times 1620$ ? $), 3280 \mathrm{MHz}$ $(2 \times 1640), 3380 \mathrm{MHz}(2 \times 1680$ ?), and $3450 \mathrm{MHz}$. 
June 6, 2000

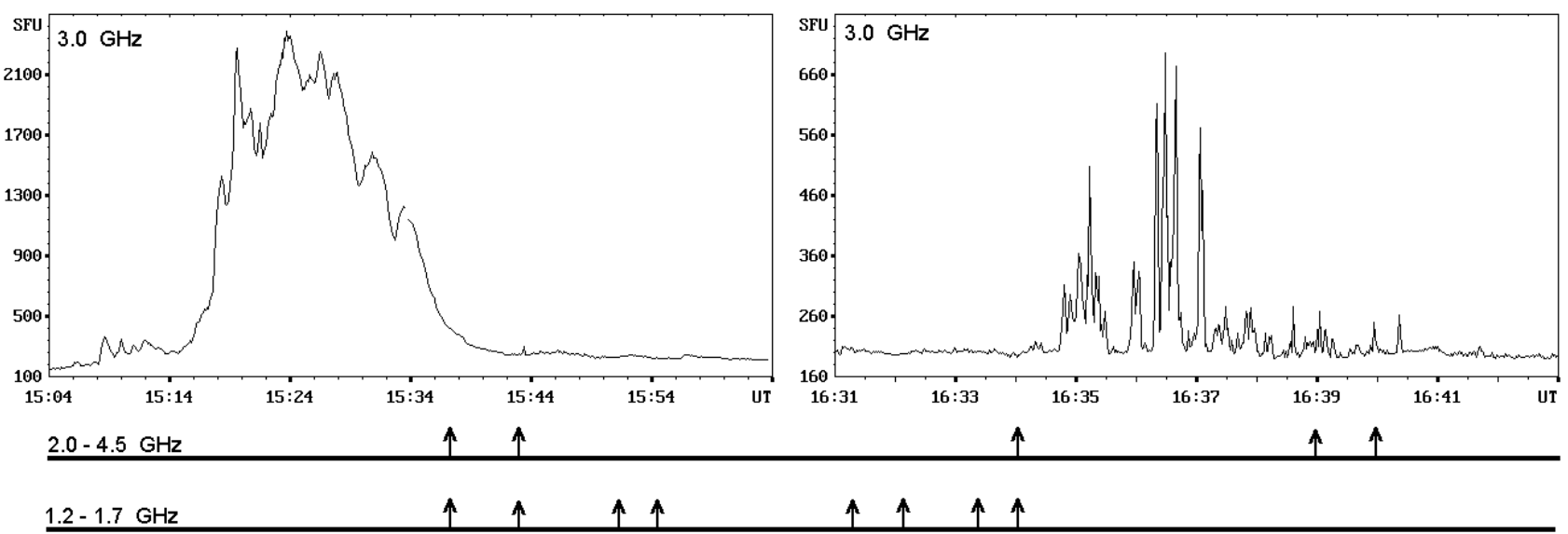

Fig. 1. The $3 \mathrm{GHz}$ radio flux record of two parts of the June 6, 2000 flare observed by the Ondřejov radiometer. The arrows indicate times of the zebra pattern observations in the $2.0-4.5 \mathrm{GHz}$ and $1.2-1.7 \mathrm{GHz}$ ranges - see also Tables 1 and 2 . Note different time and flux scales in both parts of the figure.

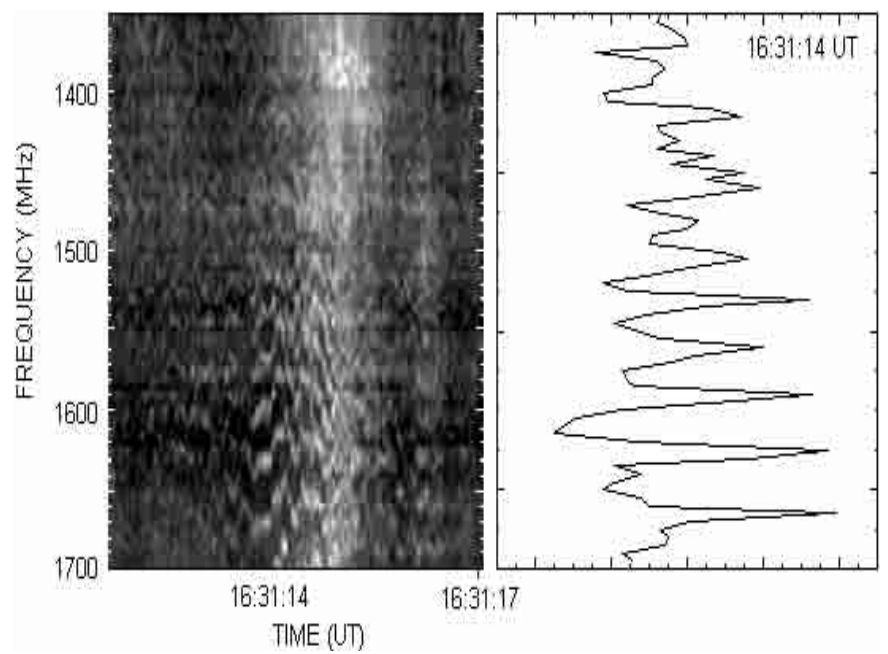

Fig. 2. An example of the $1.2-1.7 \mathrm{GHz}$ zebra pattern observed at 16:31:14 UT by the BSS, and its frequency cut.

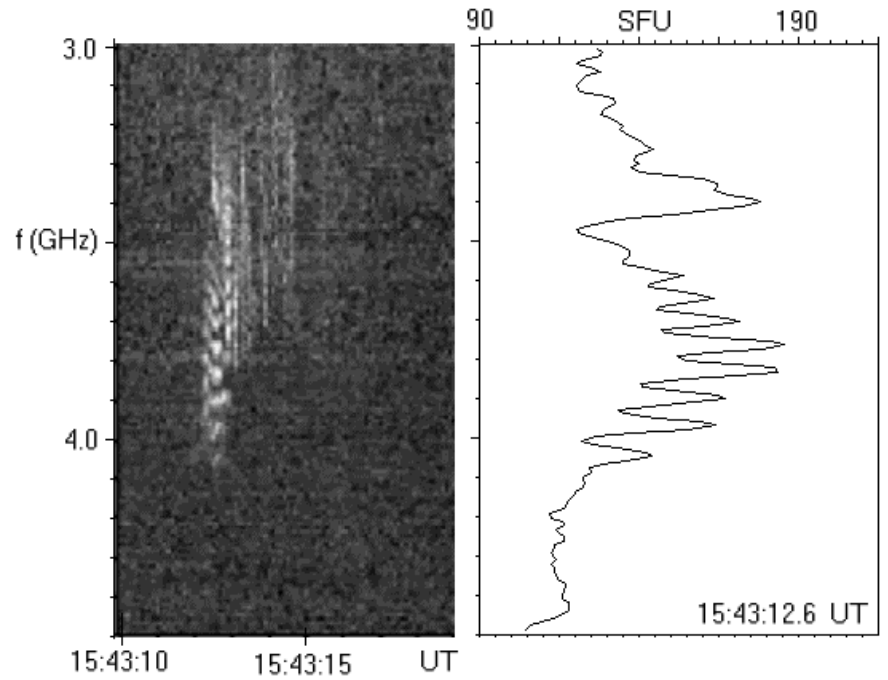

Fig. 3. An example of the $2.0-4.5 \mathrm{GHz}$ zebra pattern observed at 15:43:12 UT by the Ondřejov radiospectrograph, and its frequency cut.
Table 1. Zebra patterns in the $1.2-1.7 \mathrm{GHz}$ range.

\begin{tabular}{cccccc}
\hline \hline Zebra & $\begin{array}{c}\text { Start } \\
{[\mathrm{UT}]}\end{array}$ & $\begin{array}{c}\text { End } \\
{[\mathrm{UT}]}\end{array}$ & $\begin{array}{c}\text { Freq. range } \\
\mathrm{MHz}\end{array}$ & $\begin{array}{c}\text { No. } \\
\text { zebra } \\
\text { lines }\end{array}$ & $Q$ \\
\hline Z1 & $15: 37: 42$ & $15: 37: 50$ & $1590-1685$ & 3 & 1.9 \\
Z2 & $15: 42: 51$ & $15: 42: 52$ & $1220-1265$ & 3 & 1.6 \\
Z3 & $15: 51: 33$ & $15: 51: 34$ & $1245-1385$ & 5 & 2.7 \\
Z4 & $15: 54: 43$ & $15: 54: 47$ & $1250-1395$ & 6 & 1.4 \\
Z5 & $16: 31: 25$ & $16: 31: 27$ & $1500-1690$ & 7 & 2.5 \\
Z6 & $16: 32: 12$ & $16: 32: 25$ & $1605-1685$ & 4 & 1.7 \\
Z7 & $16: 33: 27$ & $16: 33: 34$ & $1420-1500$ & 4 & 1.4 \\
Z8 & $16: 34: 00$ & $16: 34: 06$ & $1535-1680$ & 7 & 2.2 \\
\hline
\end{tabular}

Table 2. Zebra patterns in the $2.0-4.5 \mathrm{GHz}$ range.

\begin{tabular}{cccccc}
\hline \hline Zebra & $\begin{array}{c}\text { Start } \\
{[\mathrm{UT}]}\end{array}$ & $\begin{array}{c}\text { End } \\
{[\mathrm{UT}]}\end{array}$ & $\begin{array}{c}\text { Freq. range } \\
\mathrm{MHz}\end{array}$ & $\begin{array}{c}\text { No. } \\
\text { zebra } \\
\text { lines }\end{array}$ & $Q$ \\
& & & & 2 & 1.3 \\
\hline Z9 & $15: 37: 44$ & $15: 37: 49$ & $3300-3400$ & 2 & \\
Z10 & $15: 43: 12$ & $15: 43: 13$ & $3500-4000$ & 6 & 1.2 \\
Z11 & $16: 33: 59$ & $16: 34: 02$ & $2000-2200$ & 3 & 1.5 \\
Z12 & $16: 38: 58$ & $16: 39: 00$ & $2000-2600$ & 8 & 1.4 \\
Z13 & $16: 39: 57$ & $16: 39: 59$ & $2000-2500$ & 8 & 1.6 \\
\hline
\end{tabular}

\section{The zebra patterns analysis and magnetic field strengths}

In observed zebras (Z1-Z13) we measured the ratio of frequencies of the neighboring zebra lines (numbered for the specific zebra as $1,2, \ldots$ from high to low frequencies) and the results are shown in Table 3. The frequency ratios between the neighboring zebra lines are designated as e.g. 1-2, which means the 

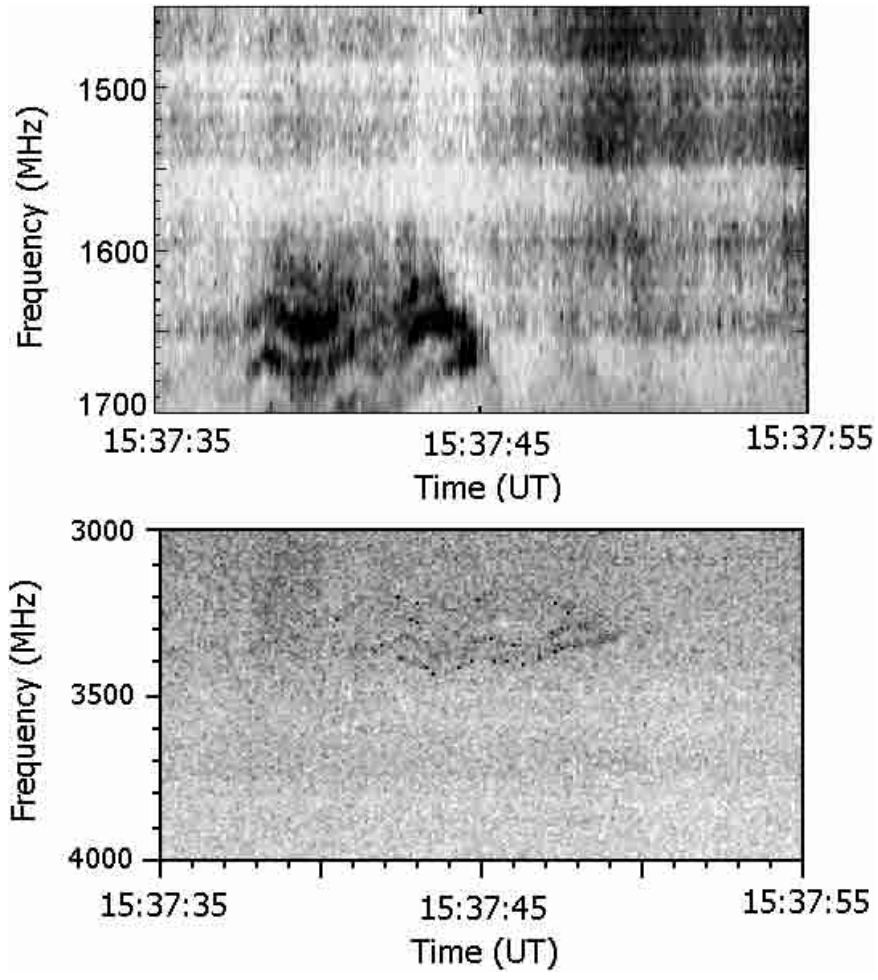

Fig. 4. The zebra pattern observed in harmonic ratio by BBS (upper) and Ondřejov (bottom) radio spectrographs. Notice the reversed color scale compared with Figs. 2 and 3.

ratio of frequencies of the zebra line 1 and 2. As can be seen these ratios are in the interval 1.009-1.037 and there is a tendency of a decrease of these ratios towards higher numbers of zebra lines, i.e. towards lower frequencies within the selected zebra. This trend was predicted in the theoretical model for the zebra pattern emission by Ledenev et al. (2001). In this model with the spatially distributed zebra line sources, in the atmosphere where the magnetic field gradient is much greater than that of the density gradient, the ratio of frequencies of the neighboring zebra lines is expressed as

$\frac{\omega_{s}}{\omega_{s+1}}=\frac{\omega_{\text {pes }}}{\omega_{\text {pes }+1}}\left[\frac{s^{3}(s+2)}{(s+1)^{3}(s-1)}\right]^{1 / 2}$,

where $\omega_{\text {pes }}$ is the electron plasma frequency at the $s$-resonance, and $s$ is the integer harmonic number. If now, due to low density gradient in zebra radio sources, we take roughly $\omega_{\text {pes }} \approx \omega_{\text {pes }+1}$ then $\omega_{2} / \omega_{3} \approx 1.088, \omega_{3} / \omega_{4} \approx 1.027, \omega_{4} / \omega_{5} \approx 1.012, \omega_{5} / \omega_{6} \approx$ 1.006 and so on. We can see that these ratios are close to those measured (Table 3). Furthermore, the Ledenev et al. model naturally explains the existence of the $\mathrm{F} / \mathrm{H}$ related branches of zebras. Namely, this plasma emission model suggests the coalescence process of the generated upper-hybrid waves with low-frequency waves for the fundamental branch $(u+l \rightarrow t$, where $t$ is the electromagnetic plasmon) and the coalescence of the upper-hybrid waves with the back-scattered ones for the harmonic branch $(u+u \rightarrow t)$. For these reasons we used the Ledenev et al. (2001) model for the following magnetic field estimations.
Table 3. Ratios of frequencies of the neighboring zebra lines for the zebras presented in Tables 1 and 2. The numbers 1 and 2 in the expression "1-2" mean numbers of zebra lines used for the ratio calculations; the zebra lines are numbered from the highest frequency towards lower frequencies, i.e. 1 means the zebra line with the highest frequency and so on. $B$ is the magnetic field in Gauss.

\begin{tabular}{cccccccc}
\hline \hline & $1-2$ & $2-3$ & $3-4$ & $4-5$ & $5-6$ & $6-7$ & $B(\mathrm{G})$ \\
\hline Z1 & 1.024 & 1.009 & & & & & 150 \\
Z2 & 1.020 & 1.016 & & & & & 113 \\
Z3 & 1.037 & 1.023 & 1.024 & 1.024 & & & 124 \\
Z4 & 1.026 & 1.015 & 1.023 & 1.023 & 1.024 & & 125 \\
Z5 & 1.021 & 1.028 & 1.019 & 1.019 & 1.016 & 1.017 & 151 \\
Z6 & 1.015 & 1.018 & 1.016 & & & & 150 \\
Z7 & 1.020 & 1.021 & 1.014 & & & & 134 \\
Z8 & 1.018 & 1.015 & 1.019 & 1.009 & 1.016 & 1.013 & 150 \\
\hline Z9 & 1.019 & & & & & & 152 \\
Z10 & 1.020 & 1.020 & 1.015 & 1.018 & 1.015 & & 178 \\
Z11 & 1.021 & 1.011 & & & & & 196 \\
Z12 & 1.030 & 1.027 & 1.037 & 1.023 & 1.024 & 1.023 & 232 \\
Z13 & 1.031 & 1.028 & 1.023 & 1.024 & 1.030 & 1.023 & 223 \\
\hline
\end{tabular}

In this estimation the most critical aspect is the determination of the $s$ harmonic numbers for the zebra lines. It can be done by a comparison of the highest frequency ratio in the specific zebra pattern with the theoretical values. In our case, the best fit is $s=4$ for the zebra line with the highest frequency. Then the magnetic field strength can be determined in the different zebra pattern sources as

$B=\frac{f_{\max }}{2.8 s}$,

where $s=4$ (for the zebra line with the highest frequency) is taken, $B$ is in Gauss and $f_{\max }$ is the highest frequency of zebra lines in MHz. Results of these estimations are shown in the last column of Table 3.

\section{Conclusion}

In this paper examples of the high-frequency zebra patterns observed by both the Brazilian Solar Spectroscope and the Ondřejov radiospectrograph during the June 6, 2000 flare are shown. One of the patterns appears at frequencies of ratio $1: 2$ (fundamental/harmonic mode of the plasma frequency). This observation favours plasma emission models of zebra pattern continuum fine structure. The intensity of harmonic branch was weaker than that of the fundamental one. The modulation depths $Q$ of zebras are 1.4-2.7 in the (1.2-1.7) GHz range and $1.2-1.6$ in the (2.0-4.5) GHz range. These values are lower than those (2.7-3.4) reported by Aurass \& Chernov (1983) for the zebras observed in the metric range. It was found that the ratio of frequencies of the neighboring zebra lines is in the range of 1.009-1.037 and there is a tendency of a decrease 
of this ratio with the decreasing frequency. (The uncertainty of $20 \mathrm{MHz}$ in frequency measurements has a negligible effect on these results.) These facts are in good agreement with the Ledenev et al. (2001) model and that is why this model was used for magnetic field estimations. The magnetic field values in the zebra radio sources were found in the range of 113$232 \mathrm{G}$.

Acknowledgements. This work was supported by the grant IAA3003202 of the Academy of Sciences of the Czech Republic. F.C.R. Fernandes acknowledge receiving scholarship from FAPESP (99/10529-0). The authors thank to the referee, Dr. H. Aurass, for helpful comments and to H. Mészárosová for technical help.

\section{References}

Aurass, H., \& Chernov, G. P. 1983, Sol. Phys., 84, 339

Chernov, G. P. 1990, Astron. Nach., 311, 419

Isliker, H., \& Benz, A. O. 1994, A\&AS, 104, 145

Jiřička, K., Karlický, M., Mészárosová, H., \& Snížek, V. 2001, A\&A, 375,243

Kuijpers, J. 1975, Thesis, Utrecht University

Ledenev, V. G., Karlický, M., Yan, Y., \& Fu, Q. 2001, Sol. Phys., 202, 71

Rosenberg, H. 1972, Cosmic Plasma Physics, ed. K. Schindler (Plenum Press, New York)

Sawant, H. S., Subramanian, K. R., Faria, C., et al. 2001, Sol. Phys., 200,167

Slottje, C. 1972, Proc. of the 2nd Meeting of the CESRA, 1971 (Trieste), 88

Zheleznakov, V. V., \& Zlotnik, E. Ya. 1975, Sol. Phys., 44, 461 